\title{
Progression of macular atrophy after PDT combined with the COX-2 inhibitor Nabumetone in the treatment of neovascular ARMD
}

\author{
Martin Sin ${ }^{\mathrm{a}}$, Oldrich Chrapek ${ }^{\mathrm{a}}$, Marta Karhanova ${ }^{\mathrm{a}}$, Zuzana Pracharovaa ${ }^{\mathrm{a}}$ Katerina Langova ${ }^{\mathrm{b}}$, Jiri Rehak ${ }^{\mathrm{a}}$
}

\begin{abstract}
Aim. To evaluate photodynamic therapy (PDT) combined with the preferential the cyclooxygenase-2 (COX-2) inhibitor, nabumetone in the treatment of the neovascular age-related macular degeneration (ARMD).

Methods. A prospective, double-blind, randomized study on 60 patients with subfoveal CNV secondary to ARMD without any previous treatment. Patients were divided into a nabumetone or placebo group. The main endpoints were the change of best-corrected visual acuity (BCVA), central macular thickness (CRT) and number of required PDT treatments. Results. In the nabumetone group, 27 patients (90\%) and 28 (93\%) in the placebo group completed the follow-up of 12 months. In the nabumetone group, the mean CRT decreased from $332 \mu \mathrm{m}$ (SD $68 \mu \mathrm{m})$ to $220 \mu \mathrm{m}$ (SD $46 \mu \mathrm{m}$ ). In the placebo group, CRT decreased from $331 \mu \mathrm{m}$ (SD $72 \mu \mathrm{m}$ ) to $254 \mu \mathrm{m}$ (SD $61 \mu \mathrm{m})$. The mean BCVA was 0.68 log MAR (SD $0.22 \log M A R$ ) in the nabumetone group and $0.62 \log$ MAR (SD $0.23 \log$ MAR) in the placebo group at baseline. This stabilised in the placebo group to $0.66 \log$ MAR (SD 0.33) but deteriorated in the nabumetone group to 0.86 logMAR (SD $0.41 \log$ MAR). There was a significant reduction in the number of required PDTs in the nabumetone group, but significant progression of the RPE atrophy area.

Conclusion. Combined PDT with oral intake of the COX-2 inhibitor, nabumetone reduced the number of required PDT retreatments, but worsening BCVA caused by macular atrophy progression. Therefore the combination of the PDT with the nabumetone is not recommended.
\end{abstract}

Key words: age-related macular degeneration, cyclooxygenase-2, CD36 metabolic pathway, combination therapy, nabumetone, macula atrophy progression, choroidal atrophy

Received: March 1, 2012; Accepted: June 20, 2012; Available online: November 6, 2012 http://dx.doi.org/10.5507/bp.2012.066

${ }^{a}$ Department of Ophthalmology, University Hospital Olomouc and Faculty of Medicine and Dentistry, Palacky University Olomouc, Czech Republic

${ }^{b}$ Department of Medical Biophysics, Faculty of Medicine and Dentistry, Institute of Molecular and Translational Medicine, Palacky University Olomouc

Corresponding author: Martin Sin, e-mail:sin.martin@seznam.cz

\section{INTRODUCTION}

Since its introduction, photodynamic therapy (PDT) has become widely used as a treatment option in patients with subfoveal choroideal neovascularisation in age-related macular degeneration (ARMD). Its treatment effects were well-established by the TAP study ${ }^{1}$. PDT uses photothrombosis to destroy vessels in choroideal neovascularisation $(\mathrm{CNV})$. This is triggered via photo-activation by the drug verteporfin administered intravenously, with subsequent production of free radicals and other oxidative metabolites leading to the destruction of the exposed endothelial cells and consequent thrombosis. Later studies showed that PDT affects the expression of pro-angiogenic and pro-inflammatory factors ${ }^{2-4}$. Recent developments in the field of anti-angiogenic treatment targeting vascular endothelial growth factor (VEGF) have changed the approach to CNV treatment and PDT is today used in combination with other treatment options such as anti-VEGF, triamcinolone, and dexamethasone ${ }^{5}$.

Cyclooxygenaese (COX) is an enzyme converting arachidonic acid to prostaglandins, prostacyclins and thromboxans. There are three main isoforms of cyclooxygenase - COX-1, COX-2 and COX-3. COX-1 is impor- tant in the normal homeostatic function of many organ systems. COX-2 expression mainly occurs in areas of inflammation ${ }^{6}$. In 2002, a new COX isoform, COX-3, was discovered. However, little is known about COX-3 function to date and there is debate over whether it is only a splicing variant of the COX-1 gene ${ }^{7}$. On the other hand, there is clear evidence of the pathogenic role of COX-2 in the angiogenesis of neovascularisation in various retinal diseases ${ }^{8}$. More recently a laboratory study was performed confirming the expression of COX-2 in $\mathrm{CNV}$ secondary to ARMD in humans?. There are a number of many potential roles for COX-2 in the pathogenesis of CNV. Cyclooxygenase-2 is involved in the amplification of VEGF function in hypoxic tissue via the prostaglandin pathway ${ }^{10}$. COX-2 expression down-regulates the endogenous anti-angiogenic factor thrombospondin-1 (TSP-1) and its receptor CD36 which interrupts angiogenesis through the p38 MAP kinase captase-3 pathways ${ }^{11}$. Moreover, COX-2 expression directly or indirectly affects production of many other growth factors (basic fibroblast growth factor, hepatocyte growth factor, epidermal growth factor, transforming growth factor beta etc.) involved in $\mathrm{CNV}$ formation ${ }^{10}$. Use of COX-2 inhibition after PDT was first described by Ferrairo et al. in 2002 for potentiating 
anti-tumor effects ${ }^{12}$. Since then, COX-2 inhibitors have been used in experimental studies that investigate their potential for suppressing neovascular growth in animal models, with promising results ${ }^{13-15}$. However clinical data in humans is still lacking. For these reasons we decided to conduct a trial using a COX-2 inhibitor in combination with PDT in the treatment of patients with CNV.

For the study, the nabumetone (Relifex, Meda AB, Solna, Sweden) as a representative of the preferential COX-2 inhibitors has been chosen. Nabumetone is a non-steroidal anti-inflammatory prodrug which exerts its pharmacological effects via the metabolite 6-methoxy2-naphthylacetic acid (6-MNA). Nabumetone itself is non-acidic and following absorption, undergoes extensive first-pass metabolism to form the main circulating active metabolite 6-MNA which is a much more potent inhibitor of COX-2. Nabumetone is clinically used mainly for the management of patients with osteoarthritis and rheumatoid arthritis to reduce pain and inflammation. Compared with COX-2 selective inhibitors, nabumetone exhibits similar anti-inflammatory and analgesic properties in patients with arthritis and there is no evidence of excessive gastrointestinal or other forms of complication to date ${ }^{16}$. We chose a preferential COX-2 inhibitor rather than a selective inhibitor owing to the lower potential cardiovascular risk of preferential inhibitors important especially in patients over sixty ${ }^{17}$.

\section{METHODS}

We performed a prospective, double-blind, randomized study comparing the effect of a combination of PDT with nabumetone and PDT with placebo. The sample consisted of 60 patients with classic or predominantly classic subfoveolar CNV secondary to ARMD without any previous treatment for ARMD. Inclusion criteria were: classic or predominantly classic CNV according to the definition by TAP study group ${ }^{1}$ secondary to ARMD confirmed by FAG, BCVA 20/200 or better, age over 50. The exclusion criteria were: $\mathrm{CNV}$ greater linear dimension larger than $5400 \mu \mathrm{m}$, use of non-steroid anti-inflammatory drug in their medical history, allergy to fluorescein or nabumetone, renal dysfunction defined as gromelural filtration rate less than $90 \mathrm{~mL} / \mathrm{min} / 1.73 \mathrm{~m}^{2}$, hepatic dysfunction defined as alanine transaminase elevation over $60 \mathrm{U} / \mathrm{L}$ and aspartate transaminase elevation over $40 \mathrm{U} / \mathrm{L}$.

Enrollment of patients was carried out consecutively between February 2008 and October 2009. The study was approved by the local ethics committee and was performed in accordance with GCP and the Declaration of Helsinki. All patients signed prior informed consent. The primary endpoints were change of best-corrected visual acuity (BCVA) measured in logarithmic minimum angle of resolution ( $\log$ MAR). Secondary endpoints were central macular thickness (CMT) measured on optical coherent tomography (OCT), the number of required PDTs during 12 months follow-up and area of macular atrophy measured at the end of follow up. Furthermore the prevalence of adverse events and suspected unexpected serious adverse reaction (SUSAR) has been evaluated in both study arms. The patients could be transfer on anti-VEGF therapy due to 2 reasons. First was worsening of BCVA more than 15 letters on ETDRS chart between two study visits, second was enlargement of CNV size over than $5400 \mu \mathrm{m}$ during the study.

During the follow-up time of 12 months the patients underwent following study visits: baseline, months 3, 6, 9 and the end of study at month 12 . In each visit following examinations were performed: BCVA investigation using the Early Treatment Diabetic Retinopathy Study charts (ETDRS), measurement of CRT using the OCT (Stratus, Carl Zeiss Meditec, Dublin, CA), fundus photography and fluorescein angiography (FAG), slit lamp investigation, intraocular pressure measurement by Goldman applanation tonometry and the biomicroscopy of the fundus. PDT with Verteporfin (Visudyne, Novartis, Basel, Switzerland) was conducted in all patients at baseline with standard parameters - 50mJ, $83 \mathrm{~s}$ and spot size 1000 $\mu \mathrm{m}$ exceeded of greater linear dimension of CNV. During subsequent examinations, PDT was done only if there was evidence of persisting leakage in the FAG as per the TAP study protocol ${ }^{1}$. After all PDTs, patients were treated with either $500 \mathrm{mg}$ of nabumetone or a placebo twice daily for the following 10 days. Randomization and masking were performed by the Department of Pharmacology of University Hospital Olomouc. We analysed the area of RPE atrophy after 12 months of follow-up. The analysis was done using a method described by Dewi et al. using a hyperflorescence area without leak in late phase of florescein angiograms ${ }^{18}$. The measurement was performed using Topcon i-base software (version 3.8.1.) The area of window defect was bordered manually by the investigator. At the end of the study, we deblinded the subjects and statistically analysed the results.

\section{Statistical methods}

Shapiro-Wilk tests were applied to test for normal distribution of the data. For normally distributed data, t-tests were used (independent or paired). The normality assumption was not satisfied for the CRT variable measurement. CRT was analyzed by non-parametric tests: Wilcoxon signed-rank test, Mann-Whitney U test, Fisher's exact test. Statistical significance was considered at $P<0.05$. SPSS v. 15 (SPSS Inc. Chicago, USA) was used.

\section{RESULTS}

In the nabumetone group, 27 patients (90\%) completed the follow-up and 3 discontinued during the follow-up. The mean patient age was 75.7 (SD 7.58) years: 14 females and 13 males completed the study. In the placebo group, 28 patients (93\%) finished the study. The mean age was 72.5 (SD 8.10). The female/male ratio was $16 / 12$ in patients who finished. Table 1 presents the demographic parameters of patient groups at baseline visit. There were no statistically significant differences between groups. 
Table 1. Patients demographics.

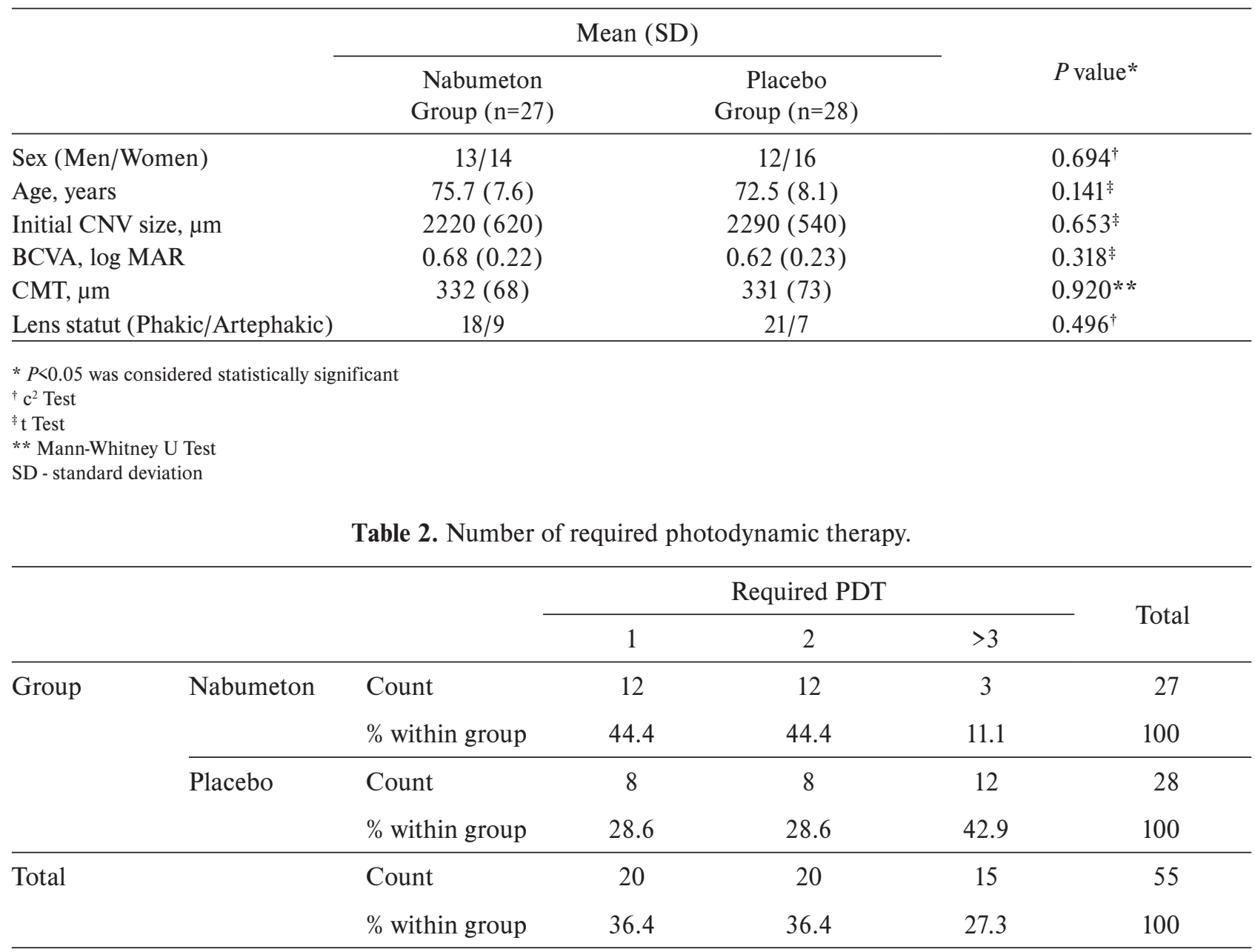

In Table 2 the number of patients requiring 1, 2, 3 or more PDTs during follow-up is shown. There was a statistically significant lower number of PDTs required in the nabumetone group ( $P=0.033$ Fisher's exact test).

We performed the OCT fast macular scan test in all subjects to evaluate CMT. Before analysis we checked all subjects and used a manual correction for exact surface and RPE bordering. We used a central $1 \mathrm{~mm}$ sub-field thickness as a reference value. The mean CMT in the nabumetone group at baseline visit was 332 (median 332) $\mu \mathrm{m}$ (SD $68 \mu \mathrm{m}$ ). This decreased to 220 (median 209) $\mu \mathrm{m}$ (SD $46 \mu \mathrm{m}$ ) at the 12 month visit. In the placebo group, the mean CMT decreased from 331(median 316) $\mu \mathrm{m}$ (SD $72 \mu \mathrm{m}$ ) to 254 (median 234) $\mu \mathrm{m}$ (SD $61 \mu \mathrm{m}$ ) during the follow-up period. There was a statistically significant reduction in both groups; nabumetone group $P<0.0001$, placebo group $P=0.0002$ (Wilcoxon signed-rank test, 2-tailed) (Fig.1).

The mean BCVA in the nabumetone group decreased from $0.68 \log$ MAR (SD 0.22 log MAR) at baseline to $0.86 \log$ MAR (SD 0.41 log MAR) at moths 12. There was a statistically significant reduction in BCVA; $P=0.008$ (paired t-test, 2-tailed). The mean BCVA in the placebo group at baseline was $0.62 \log$ MAR (SD $0.23 \log$ MAR) and did not significantly changed compared with month 12 (0.66 log MAR;SD 0.33; $P=0.43$ ) (Fig. 2).
In the placebo group, the mean area in month 12 was $3.63 \mathrm{~mm}^{2}$ (SD $1.42 \mathrm{~mm}^{2}$ ) and $5.28 \mathrm{~mm}^{2}$ (SD 2.33 $\mathrm{mm}^{2}$ ) in the nabumetone group. There was a significant difference between groups in atrophy size at month $12 ; P=0.003$ (t-test for equality of means) (Fig.3)

Five patients had a lost of follow-up, 3 in the nabumetone group and 2 in the placebo group. One patient discontinued the study for AE related to nabumetone: a male aged 76 years who experienced nausea after nabumetone administration. He was withdrawn from the study 3 days after randomization. One patient in the placebo group discontinued after month 6 for personal reasons and 3 more ( 2 from the nabumetone group, and 1 from the placebo group) were transferred to anti VEGF therapy based on the reason defined on the begining of the study. In one case it was a worsening of BCVA betwen consecutive visits and in 2 cases it was enlargement of CNV size over $5400 \mu \mathrm{m}$. None of these patients were used for the final data evaluation. No patient underwent any ocualr surgery.

\section{DISCUSSION}

Our results showed the reduction of the number of PDTs when PDT is combined with nabumetone but this 


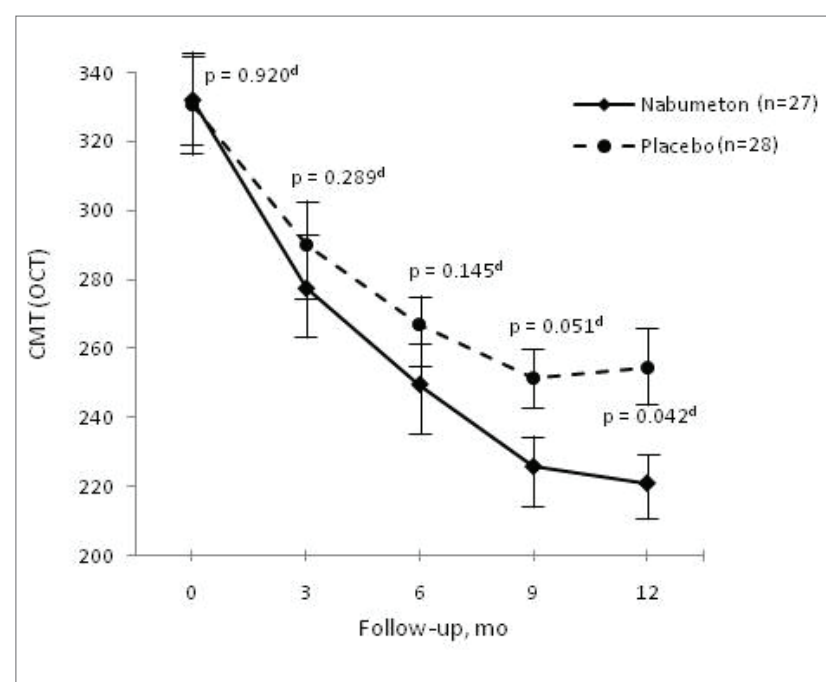

Fig. 1. Mean central macular thickness resulted in $\mu \mathrm{m}$ during follow-up. The standard error of the mean (SE) is shown.

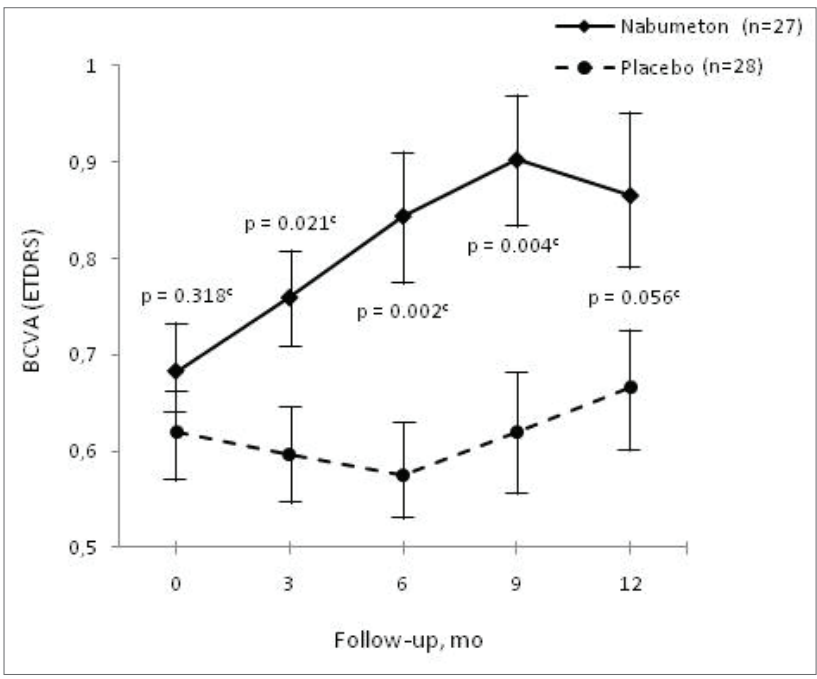

Fig. 2. Mean best-corrected visual acuity in logMAR during follow-up. The standard error of the mean (SE) is shown.

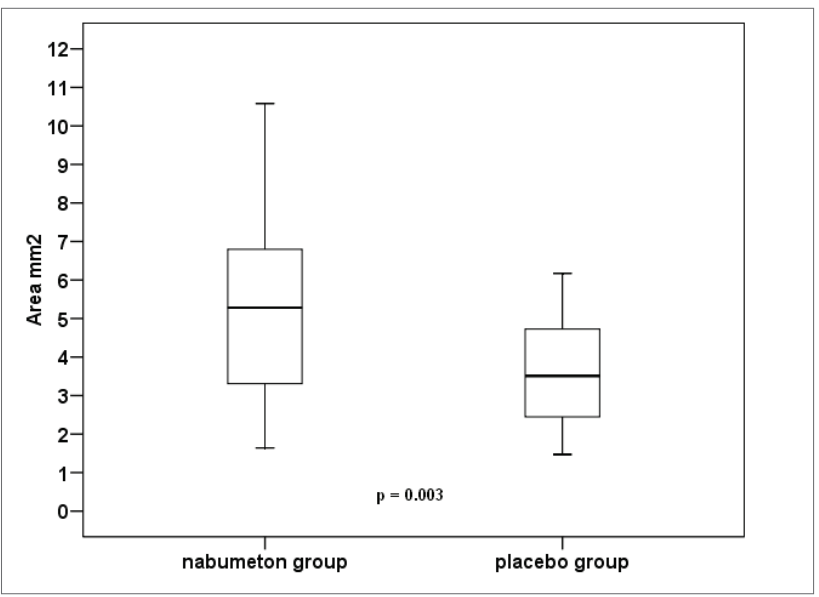

Fig. 3. Box graf showing a difference of RPE atrophy area after 12 month of follow-up. was followed by a significant worsening of BCVA compared to PDT and placebo. Though studies comparing PDT and anti-VEGF therapy show the greater efficacy of anti-VEGF therapy ${ }^{19}$, there are still a number of patients who do not respond to anti-VEGF therapy ${ }^{20}$. These patients could potentially benefit from PDT in combination with other treatment options than anti-VEGF therapy.

To our knowledge, this is the first study evaluating the combination of PDT and the oral preferential COX-2 inhibitor in the treatment of wet ARMD. The short-term effect of adjunctive topical diclofenac was described in the ADD-V study ${ }^{21}$. This study investigating 61 patients was published in 2007 and showed no statistical difference between the placebo and the diclofenac group. The limitation of this study was a short follow-up time of 3 month's. It remains questionable whether eye drops can penetrate to the posterior pole of the eye. The effect of acetylsalicid acid (a non-selective COX inhibitor) adjustment to PDT appears to be negative. Ranchod et al. retrospectively analyzed 244 eyes of patients using aspirin who had been treated with PDT and found a statistically significant worsening in VA and increased need for PDTs $\left(\right.$ ref. $\left.^{22}\right)$. The negative effect of non-selective COX inhibitors was explained by decrease in platelet function due to COX-1 metabolism ${ }^{23}$. However this does not explain our results apropos reduction in PDTs for the nabumetone group. Our results correspond with another study showing nabumetone has only minor effects on platelet function ${ }^{24}$.

The unexpected worsening in BCVA in the nabumetone group at the end of follow-up can be explained, in our opinion, by the progression of atrophic changes in the macular region: there was a statistically significant larger area of RPE atrophy after nabumetone treatment. Dewi et al. suggest that RPE atrophy is the natural course of CNV development ${ }^{18}$. Unfortunately, due to the absence of pretreatment evaluation of RPE this finding is limited. However, oedema and hemorrhage are ruled out as causative. The two groups had comparable parameters at the outset and hence the size of the RPE atrophy should have been roughly the same at the end. In addition, the slightly and statistically insignificant, greater decrease in retinal thickness in the nabumetone group could be considered to be the result of atrophy. We used the time domain OCT with its technological limits compared to spectral domain OCT which may have been potentially more practical in terms of evaluation.

Our clinical observations support the role of COX-2 down-regulation in choroidal involution progression hypothesized by Houssier et al. ${ }^{25}$. These authors discovered the role of the CD36 receptor and COX-2 in the progression of atrophic ARMD using rodent models. It has been suggested that the underlying condition is oxidative stress which is greatly increased during PDT. The question remains about the role of VEGF in this molecular process. VEGF is suppressed by COX-2 inhibition. There is clear evidence of the neuroprotective effect of VEGF on retinal cells after hypoxia as well ${ }^{26}$. These studies emphasise the crucial role of VEGF in hypoxia not only for vessel proliferation but for neuronal survival. PDT leads 
to sudden hypoxia and changes in the microenvironment of the macular region ${ }^{2-4}$. These changes are important for initiating vessel growth to restore normal oxygen levels in a hypoxic macular region and secondarily for protecting the extremely vulnerable neuronal cells. VEGF suppression potentially leads to atrophy progression. On the other hand, the initial results of the combination of PDT with anti-VEGF look promising ${ }^{5}$. The exact role of VEGF in the progression of the dry form of ARMD remains to be elucidated in future studies. It is highly probably that there are other currently undiscovered mechanisms in action. In our opinion nabumetone is a double-edged sword in ARMD treatment. Reduction in number of required PDTs confirm its anti-angiogenic effect.

The limitations of this study include small sample size and only a one year follow-up. Some measurements were made subjectively (RPE atrophy area bordering and OCT thickness bordering) and this may have led to bias. The question remains whether a preferential COX-2 inhibitor has the same (positive or negative) effect as a selective COX-2 inhibitor. The prospective design and doubleblinding were advantages. Our results have uncovered a potential limitation in the use of nabumetone in combination with PDT. The clinical effect of this therapy needs to be evaluated in future studies for better understanding of the many still unknown mechanisms of COX-2 in ARMD pathogenesis.

\section{CONCLUSIONS}

The combination of photodynamic therapy with oral intake of the preferential COX-2 inhibitor nabumetone showed a significant decrease in number of PDT repetitions required in a one year of follow-up compared to PDT and placebo. This was followed however, by a worsening of BCVA in the nabumetone group and demonstrated macular atrophy progression. These results suggest a potential limit to the combination of nabumetone with PDT.

\section{ACKNOWLEDGEMENTS}

This work was supported by Grant Project CZ.1.05/2.1.00/01.0030

The study was approved by the ethics committee of University Hospital Olomouc - reference number 132/07.

\section{CONFLICT OF INTEREST STATEMENT}

The authors stated that there are no conflicts of interest regarding the publication of this article.

\section{REFERENCES}

1. Treatment of age-related macular degeneration with photodynamic therapy (TAP) study group: photodynamic therapy of subfoveal choroidal neovascularization in age-related macular degeneration with verteporfin: one-year results of 2 randomized clinical trials. TAP report 1. Arch Ophthalmol 1999;117:1329-45.

2. Schmidt-Erfurth U, Schlötzer-Schrehard U, Cursiefen C, Michels S, Beckendorf A, Naumann GO. Influence of photodynamic therapy on expression of vascular endothelial growth factor (VEGF), VEGF receptor 3 , and pigment epithelium-derived factor. Invest Ophthalmol Vis Sci 2003;44:4473-80.

3. Tatar O, Adam A, Shinoda K, Stalmans P, Eckardt C, Lüke M, BartzSchmidt KU, Grisanti S. Expression of VEGF and PEDF in choroidal neovascular membranes following verteporfin photodynamic therapy. Am J Ophthalmol 2006;142:95-104.

4. Tatar O, Shinoda K, Adam A, Eckert T, Eckardt C, Lucke K, Deuter C, Bartz-Schmidt KU, Grisanti S. Effect of verteporfin photodynamic therapy on endostatin and angiogenesis in human choroidal neovascular membranes. Br J Ophthalmol 2007;91:166-73.

5. Shah GK, Sang DN, Hughes MS. Verteporfin combination regimens in the treatment of neovascular age-related macular degeneration. Retina 2009;29:133-48.

6. Rajakariar R, Yaqoob MM, Gilroy DW. COX-2 in inflammation and resolution. Mol Interv 2006;6:199-207.

7. Davies NM, Good RL, Roupe KA, Yáñez JA. Cyclooxygenase-3: axiom dogma, anomaly, enigma or splice error? - Not as easy as 1, 2, 3. J Pharm Pharm Sci 2004;7:217-26.

8. Wilkinson-Berka JL. Vasoactive factors and diabetic retinopathy: vascular endothelial growth factor, cycoloxygenase-2 and nitric oxide. Curr Pharm Des 2004;10:3331-48.

9. Maloney SC, Fernandes BF, Castiglione E, Antecka E, Martins C, Marshall JC, Di Cesare S, Logan P, Burnier MN Jr. Expression of cyclooxygenase-2 in choroidal neovascular membranes from age-related macular degeneration patiens. Retina 2009;29:176-80.

10. Hu W, Criswell MH, Ottlecz A, Cornell TL, Danis RP, Lambrou GN, Ciulla TA. Oral administration of lumiracoxib reduces choroidal neovascular membrane development in the rat laser-trauma model. Retina 2005;25:1054-64.

11. Chan CK, Pham LN, Zhou J, Spee C, Ryan SJ, Hinton DR. Differential expression of pro- and antiangiogenic factors in mouse straindependent hypoxia-induced retinal neovascularization. Lab Invest 2005;85:721-33.

12. Ferrario A, Von Tiehl K, Wong S, Luna M, Gomer CJ. Cyclooxygenase-2 inhibitor treatment enhances photodynamic therapy-mediated tumor response. Cancer Res 2002;62:3956-61.

13. Castro MR, Lutz D, Edelman JL. Effect of COX inhibitors on VEGFinduced retinal vascular leakage and experimental corneal and choroidal neovascularization. Exp Eye Res 2004;79:275-85.

14. Wilkinson-Berka JL, Alousis NS, Kelly DJ, Gilbert RE. COX-2 inhibition and retinal angiogenesis in a mouse model of retinopathy of prematurity. Invest Ophthalmol Vis Sci 2003;44:974-9.

15. Takahashi H, Yanagi Y, Tamaki Y, Uchida S, Muranaka K. COX2-selective inhibitor, etodolac, suppresses choroidal neovascularization in a mice model. Biochem Biophys Res Commun 2004;325(2):461-6.

16. Hedner T, Samulesson O, Währborg P, Wadenvik H, Ung KA, Ekbom A. Nabumetone: therapeutic use and safety profile in the management of osteoarthritis and rheumatoid arthritis. Drugs 2004;64:2315-43.

17. Chen YF, Jobanputra P, Barton P, Bryan S, Fry-Smith A, Harris G, Taylor RS. Cyclooxygenase-2 selective non-steroidal anti-inflammatory drugs (etodolac, meloxicam, celecoxib, rofecoxib, etoricoxib, valdecoxib and lumiracoxib) for osteoarthritis and rheumatoid arthritis: a systematic review and economic evaluation. Health Technol Assess 2008:12:1-278.

18. Dewi NA, Yuzawa M, Tochigi K, Kawamura A, Mori R. Effects of photodynamic therapy on the choriocapillaris and retinal pigment epithelium in the irradiated area. Jpn J Ophthalmol 2008;52(4):277-81.

19. Brown DM, Kaiser PK, Michels M, Soubrane G, Heier JS, Kim RY, Sy JP, Schneider S; ANCHOR Study Group. Ranibizumab versus verteporfin for neovascular age-related macular degeneration. N Engl J Med 2006;355:1432-44.

20. Lux A, Llacer $H$, Heussen FM, Joussen AM. Non-responders to bevacizumab (Avastin) therapy of choroidal neovascular lesions. $\mathrm{Br} J$ Ophthalmol 2007;91:1318-22.

21. Adjunctive diclofenac with verteporfin (ADD-V) study group. Effect of adjunctive diclofenac with verteporfin therapy to treat choroval neovascularisation due to age-related macular degeneration. Phase Il study. Retina 2007;27:693-700. 
22. Ranchod TM, Guercio JR, Ying GS, Brucker AJ, Stoltz RA. Effect of aspirin therapy on photodynamic therapy with verteporfin for choroidal neovascularization. Retina 2008;28:711-6.

23. Debefve E, Pegaz B, Ballini JP, Konan YN, van den Bergh $\mathrm{H}$. Combination therapy using aspirin-enhanced photodynamic selective drug delivery. Vascul Pharmacol 2007;46:171-80.

24. van Kraaij DJ, Hovestad-Witterland AH, de Metz M, Vollaard EJ. A comparison of the effects of nabumetone vs meloxicam on serum thromboxane B2 and platelet function in healthy volunteers. $\mathrm{Br} J$ Pharmacol 2002;53:644-647.
25. Houssier M, Raoul W, Lavalette S, Keller N, Guillonneau X, Baragatti B, Jonet L, Jeanny JC, Behar-Cohen F, Coceani F, Scherman D, Lachapelle P, Ong H, Chemtob S, Sennlaub F. CD36 Deficiency Leads to Choroidal Involution via COX2 Down-Regulation in Rodents. PLoS Med 5(2):e39. doi:10.1371/journal.pmed.0050039

26. Nishijima K, Ng YS, Zhong L, Bradley J, Schubert W, Jo N, Akita J, Samuelsson SJ, Robinson GS, Adamis AP, Shima DT. Vascular endothelial growth factor-A is a survival factor for retinal neurons and a critical neuroprotectant during the adaptive response to ischemic injury. Am J Pathol 2007;171:53-67. 